\title{
Protection Against Myocardial Dysfunction After a Brief Ischemic Period in Transgenic Mice Expressing Inducible Heat Shock Protein $\mathbf{7 0}$
}

\author{
Susanne U. Trost, ${ }^{\star}$ Jeffrey H. Omens, ${ }^{\ddagger}$ William J. Karlon, ${ }^{\ddagger}$ Markus Meyer, ${ }^{\star}$ Ruben Mestril, ${ }^{\star}$ James W. Covell, ${ }^{\ddagger}$ \\ and Wolfgang $H$. Dillmann* \\ $*$ Division of Endocrinology \& Metabolism, and ${ }^{\ddagger}$ Division of Cardiology, Department of Medicine, University of California, San Diego, \\ La Jolla, California 92093-0618
}

\begin{abstract}
Brief ischemic periods lead to myocardial dysfunction without myocardial infarction. It has been shown that expression of inducible HSP70 in hearts of transgenic mice leads to decreased infarct size, but it remains unclear if HSP70 can also protect against myocardial dysfunction after brief ischemia. To investigate this question, we developed a mouse model in which regional myocardial function can be measured before and after a temporary ischemic event in vivo. In addition, myocardial function was determined after brief episodes of global ischemia in an isolated Langendorff heart. HSP70-positive mice and transgene negative littermates underwent $8 \mathrm{~min}$ of regional myocardial ischemia created by occlusion of the left descending coronary artery, followed by $60 \mathrm{~min}$ of reperfusion. This procedure did not result in a myocardial infarction. Regional epicardial strain was used as a sensitive indicator for changes in myocardial function after cardiac ischemia. Maximum principal strain was significantly greater in HSP70-positive mice with $88 \pm 6 \%$ of preischemic values vs. $58 \pm 6 \%$ in transgene-negative mice $(P<0.05)$. Similarly, in isolated Langendorff perfused hearts of HSP70-positive and transgene-negative littermates exposed to $10 \mathrm{~min}$ of global ischemia and $90 \mathrm{~min}$ of reperfusion, HSP70 transgenic hearts showed a betterpreserved ventricular peak systolic pressure. Thus, we conclude that expression of HSP70 protects against postischemic myocardial dysfunction as shown by better preserved myocardial function. (J. Clin. Invest. 1998. 101: 855862.) Key words: heat shock protein - myocardial ischemia • myocardial function $\bullet$ transgenic animal • strain
\end{abstract}

\section{Introduction}

Brief periods of ischemia can cause reduced cardiac contractility without myocyte necrosis (1-3). Postischemic myocardial dysfunction diminishes the benefits of reperfusion in treatment of myocardial infarcts, and complicates cardiac surgery and

Address correspondence to Dr. Wolfgang H. Dillmann, University of California, San Diego, 9500 Gilman Drive, La Jolla, CA 920930618. Phone: 619-534-9934; FAX: 619-534-9932; E-mail: wdillman@ ucsd.edu

Received for publication 31 March 1997 and accepted in revised form 29 December 1997.

J. Clin. Invest.

(C) The American Society for Clinical Investigation, Inc. 0021-9738/98/02/0855/08 \$2.00

Volume 101, Number 4, February 1998, 855-862

http://www.jci.org cardiac transplantation (4). Finding ways to protect the heart from postischemic myocardial dysfunction would therefore be highly desirable. Previous studies using a myocardial stunning model in pigs suggested a correlation between elevated heat shock protein 70 (HSP70) $)^{1}$ levels induced by prior ischemic episodes and improved myocardial function after subsequent ischemia (5). Heat shock proteins can be induced by brief episodes of several stresses including ischemia (6-8), heat shock $(9,10)$, or exercise (11). However, these measures lead to changes in expression of a wide spectrum of proteins related to cellular defense mechanisms, such as oxygen-free radical scavenging enzymes $(12,13)$ and increases in several members of the heat shock protein family (14). Thus, in the setting of heat shock or other stresses, the protective effect of one specific heat shock protein such as HSP70 is difficult to discern. In transgenic mice constitutively expressing the inducible rat HSP70 in cardiac myocytes, experiments can be performed to elucidate the protective effect of HSP70 independently of prior stress situations. These HSP70-expressing transgenic mice have been successfully used to demonstrate a decrease in infarct size after $20 \mathrm{~min}$ of global and $30 \mathrm{~min}$ of regional (15, 16) ischemia. The subcellular mechanisms leading to decreased cardiac contractility after brief ischemic episodes are different from those underlying myocardial infarction. One important factor leading to postischemic myocardial dysfunction is the formation of free oxygen radicals (17-19) that may induce malformation of specific cardiac proteins (20). As HSP70 is known to protect proteins from malfolding, we wanted to determine if transgenic mice that constitutively express inducible HSP70 in their cardiac myocytes are better protected from regional myocardial dysfunction induced by brief episodes of ischemia. Therefore, we established a new technique for in vivo measurement of regional myocardial function in mice based on measurement of a two-dimensional (2-D) epicardial strain $(21,22)$. With this model, we were able to observe that the postischemic decrement in regional myocardial function is significantly less in HSP70-positive mice than in controls. The protective effect of HSP70 was confirmed in an isolated perfused Langendorff preparation in which myocardial function was assessed by ventricular peak systolic pressure. Our results in an in vivo model of regional ischemia and in an isolated perfused heart preparation undergoing global ischemia show that expression of inducible HSP70 can ameliorate myocardial dysfunction induced by a short period of ischemia.
1. Abbreviations used in this paper: $2-\mathrm{D}$, two-dimensional; CK, creatine phosphokinase; $\mathrm{E}_{1}$, maximum principal strain; $\mathrm{E}_{2}$, minimal principal strain; HSP, heat shock protein; TTC, 2,3,5-triphenyl-tetrazolium chloride. 


\section{Methods}

All experiments in this study were performed in accordance with the guidelines established by the Committee on Animal Research at the University of California, San Diego. As previously described, transgenic mice were generated by use of a chimeric transgene consisting of the rat inducible HSP70 cDNA (23) inserted in the pCAGGsS vector. The transgene is under the control of the human cytomegalovirus enhancer and chicken $\beta$-actin promoter (15), leading to well-documented overexpression of HSP70 mRNA and protein levels in the myocardium $(15,16)$.

Protein analysis. 2-D gel electrophoresis was carried out on an Immobiline drystrip ( $\mathrm{pH}$ 4.0-7.0) and an Excel SDS gel (gradient 8-18\%; Pharmacia LKB Biotechnology, Piscataway, NJ). Approximately $100 \mathrm{mg}$ of each protein sample was isoelectrically focused on an Immobiline drystrip ( $\mathrm{pH} 4.0-7.0$ ), followed by protein size separation on the Excel SDS gel according to the manufacturer's instructions. Subsequently, the 2-D gels were electrotransferred onto nitrocellulose using a semidry electrotransfer apparatus (Bio-Rad Laboratories, Hercules, CA). The nitrocellulose blots were reacted with a polyclonal antibody that binds specifically to both the mammalian inducible and constitutive HSP70 isoforms (7). Western blots were then reacted with an anti-rabbit IgG biotinstreptavidin, horseradish peroxidase-conjugated system (Vectastain, ABC kit; Vector Laboratories, Inc., Burlingame, CA), and developed with diaminobenzidine tetrahydrochloride (DAB kit; Vector Laboratories, Inc.).

Surgical preparation for in vivo study. Mice were anesthetized with Ketamine $(100 \mathrm{mg} / \mathrm{kg})$ and Xylazine $(8 \mathrm{mg} / \mathrm{kg})$. They were tracheostomized and ventilated with a Harvard rodent ventilator with room air at a rate of $100 / \mathrm{min}$ and a tidal volume of $\sim 0.5 \mathrm{ml}$, which was adjusted individually according to thorax expansion. The heart was exposed with a left thoracotomy. For better exposure of the heart surface, the fourth and fifth ribs were removed, and the pericardium was opened. An 8-0 nylon suture (Ethicon, Somerville, NJ) was placed around the proximal left anterior descending coronary artery (LAD) by entering the left ventricular wall just inferior to the left atrial appendage and exiting from the right ventricular outflow tract. The suture and a small piece of polyethylene tubing were kept in place as a reversible snare occluder. Nontoxic white titanium oxide powder (Sigma Chemical Co., St. Louis, MO) was used to create surface markers (diameter $0.02-0.04 \mathrm{~mm}$ ) on the exposed left ventricular epicardium (Fig. 1; 21). To protect the heart surface from drying, it was covered with wet tissue between measurements. The left carotid artery was isolated, and a heat-stretched fluid-filled polyethylene tubing (PE240) connected to a Bio-Tec pressure transducer (BT70; Bio Tec, San Diego, CA) was inserted (24). This cannula was also used to heparinize the mice ( $7.5 \mathrm{U}$ heparin $/ 30 \mathrm{~g}$ mouse). Four limb leads were placed subcutaneously to obtain the ECG. Body temperature was monitored with a rectal probe and kept between $36.5-37.0^{\circ} \mathrm{C}$ using a heating pad under the animal.

Data acquisition. The motion of the surface markers over several heart cycles was recorded on standard VHS video tape with a black and white video camera (High Performance CCD camera; Cohu Electronics Inc., San Diego, CA) and a 60-mm macro lens. The zoom level was such that the heart occupied the entire video field. ECG, arterial pressure, and a $60-\mathrm{Hz}$ synchronizing signal were acquired with an analog-to-digital conversion at $2,000 \mathrm{~Hz}$ on an IBM-compatible 486 computer using Windaq software (Data Instruments, Akron, $\mathrm{OH})$. These signals were synchronized to the video tape, which contained a dubbed time code for frame counting. The video recordings were captured on a Macintosh-based image analysis system (Premier; Adobe Systems Inc., Mountain View, CA). By separating one video frame in two video fields, a temporal resolution of $60 \mathrm{frames} / \mathrm{s}$ could be achieved. Each field consisted of $640 \times 240$ pixels. The titanium oxide markers appeared on the video as bright white dots ranging from 5 to 15 pixels in diameter. Three dots in the ischemic area were chosen. The centroid of each marker was determined in each video field with image analysis software (Image 1.57; National Institutes of

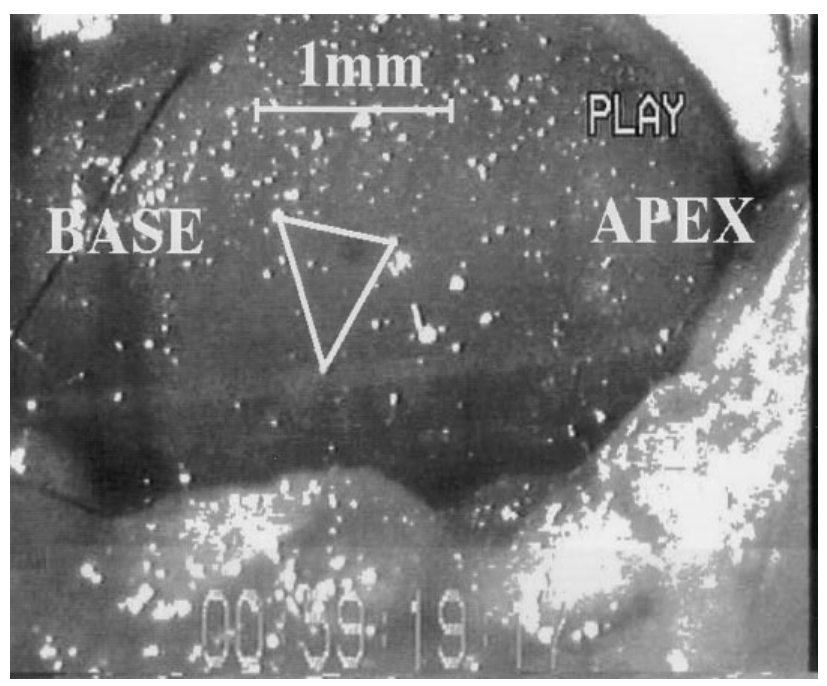

Figure 1. Original video frame of the open-chest mouse heart during data acquisition. The heart was exposed by a left thoracotomy, and the fourth and fifth ribs were removed. The bright white dots on the epicardium are titanium oxide markers, and the triangle of dots used for the strain measurements is shown.

Health), and lengths of the three sides of the triangle were computed. The two-dimensional reference coordinate system was defined at the center of the triangle in the epicardial tangent plane with the longitudinal axis parallel to the apex-aortic valve line. Lagrangian strains were calculated from the deformation of the triangle, assuming a homogenous deformation within the area of the triangle (21). The strain in the triangle was determined with respect to the enddiastolic reference configuration, and followed over several cardiac cycles without ventilation. By solving the 2-D eigen-value problem, maximum principal strain $\left(\mathrm{E}_{1}\right)$ and its associated direction (principal angle relative to circumferential) were determined. End-diastole was taken as the first video field after the QRS complex and peak systole was defined as the field with the greatest principal strain occurring in each heart cycle. The peak systolic strain tensor and principal values were found for several consecutive cardiac cycles and averaged.

Experimental protocol. In the sham group, four HSP70-positive and three transgene-negative mice underwent the surgical procedures described above, except that the snare occluder was left open. Regional myocardial function and physiologic parameters were obtained over $60 \mathrm{~min}$.

HSP70-positive mice $(n=11)$ and their transgene-negative littermates $(n=11)$ underwent 8 min of coronary artery occlusion and 60 min of reperfusion. The transgene status of the mouse was not known by the investigator during surgery and data acquisition. Time from introduction of anesthesia to the first data recording was $\sim 45 \mathrm{~min}$. Functional measurements were obtained in both groups before ischemia and after 2, 15,30, 45, and $60 \mathrm{~min}$ of reperfusion. Five mice were reperfused for an additional $30 \mathrm{~min}$ for a total of $90 \mathrm{~min}$. At the end of the protocol (after 60 or 90 min of reperfusion) the coronary artery was reoccluded, and the area of risk was demarcated by injecting 0.2 $\mathrm{ml} 0.05 \%$ Monastral Blue dye (Sigma Chemical Co.) into the apex of the left ventricle (16). The heart was excised, cut transverse to the long axis in five slices, and incubated in 2,3,5-triphenyl-tetrazoliumchloride (TTC; Sigma Chemical Co.) for 12 min to verify the absence of infarcted tissue. For histological examination of the myocardial tissue, an additional set of four transgene-negative mice were exposed to the same protocol with 8 -min ischemia and 60-min reperfusion in an open-chest preparation. After closure of the chest, the animals were extubated and regained consciousness. $5 \mathrm{~d}$ after surgery the 
mice were killed, and the hearts were fixed in $10 \%$ formalin and paraffin-imbedded. Hearts were cut in $10-\mu \mathrm{m}$ transverse sections and stained with hematoxylin and eosin or picrosirius red. 15 mice were not included in the analysis for the following reasons: one mouse was excluded because of a malfunction of the video recorder; eight mice were excluded due to problems with the surgical preparation; one transgene-negative mouse developed ventricular arrhythmia during reperfusion; two HSP70-positive and two transgene-negative mice died during reperfusion after a substantial decrease of blood pressure; one sham-operated transgene negative mouse also died after a drop in blood pressure during the protocol.

Langendorff method. Influence of brief ischemia on isolated hearts from HSP70 mice and their transgene-negative littermates was investigated in a Langendorff retrograde perfusion setup. Hearts were rapidly removed from anesthetized mice and immersed in iced hyperkalemic buffer solution ( $\mathrm{KCl}$ concentration of $9.4 \mathrm{mM}$ ), which immediately stopped contractile activity. After trimming extraneous tissue from the base of the heart and exposing aorta, a stainless steel catheter was inserted into the aortic stump, and retrograde perfusion was initiated. The time from removal of the heart to perfusion was 1-2 min. The heart immediately started to contract. The perfusion system used was similar to those previously published $(25,26)$. The perfusate, a modified Krebs-Henseleit buffer (in $\mathrm{mM}: \mathrm{NaCl}, 118 ; \mathrm{KCl}$, 4.7; $\mathrm{CaCl}_{2}, 2.25 ; \mathrm{MgSO}_{4}, 1.2 ; \mathrm{KH}_{2} \mathrm{PO}_{4}, 1.2 ; \mathrm{NaHCO}_{3}, 25.0 ; \mathrm{Na}_{2}$ EDTA, 0.5 ; and glucose, 5.5) was kept at $37^{\circ} \mathrm{C}$ and bubbled with $95 \% \mathrm{O}_{2} / 5 \%$ $\mathrm{CO}_{2}$. Perfusion pressure was maintained at $100 \mathrm{mmHg}$. After removing the left atrium, a small plastic balloon (Atane Film; Dow Corning, Midland, MI) was inserted into the left ventricular cavity. The balloon was coupled to a microsyringe and a pressure transducer (Millar Instruments, Houston, TX). The balloon was filled with water and inflated until the diastolic pressure reached $10 \mathrm{mmHg}$. Platinum wires were placed on the surface of the right atrium using a micromanipulator to pace the heart at 400 beats/min. Hearts were perfused for 15 min before data collection. Digitized recordings were made of the ventricular pressure and the stimulation pulse on an IBM-compatible 486 computer. Peak systolic pressure and end-diastolic pressure were taken from the recordings. At the end of the protocol, heart tissue was stained with TTC to verify viable tissue. An additional series of experiments were conducted to determine if $10 \mathrm{~min}$ of ischemia causes infarction or myocyte necrosis. Three groups of animals were examined: a negative control group not exposed to ischemia, a 10-min ischemia group, and a positive control group with a 30-min ischemic episode. Four animals were studied in each group. Hearts were isolated and perfused as described above. Effluent from hearts was collected for 1 min immediately before the ischemia, and at 3,30, and 60 min during the following reperfusion. The effluent was analyzed for creatine phosphokinase (CK) content using an optical densimetric assay (Procedure No. 45-UV; Sigma Chemical Co.).

Statistical analysis. Data are presented as mean \pm SEM. The statistical significance of the change in strain or peak systolic pressure over time, and the effect of the transgene status on this change were tested by ANOVA for repeated measures (SuperANOVA; Abacus Concepts Inc., Berkeley, CA). When ANOVA for repeated measures indicated statistical significance, a closed-test procedure was applied for post-hoc analysis (27). A value of $P<0.05$ was accepted as statistically significant. Differences in CK release between groups were tested with ANOVA for repeated measures and the Tukey-Kramer post hoc test.

\section{Results}

Baseline characteristics of transgene-positive and -negative mice. HSP70-positive mice were of unremarkable appearance and behavior. Body wt of HSP70-positive mice was $28.4 \pm 2.7 \mathrm{~g}$, and heart weight was $116 \pm 7 \mathrm{mg}$. These values did not differ from those of transgene-negative littermates with a body wt and heart wt of $25.8 \pm 1.8 \mathrm{~g}$ and $112 \pm 7 \mathrm{mg}$, respectively. The
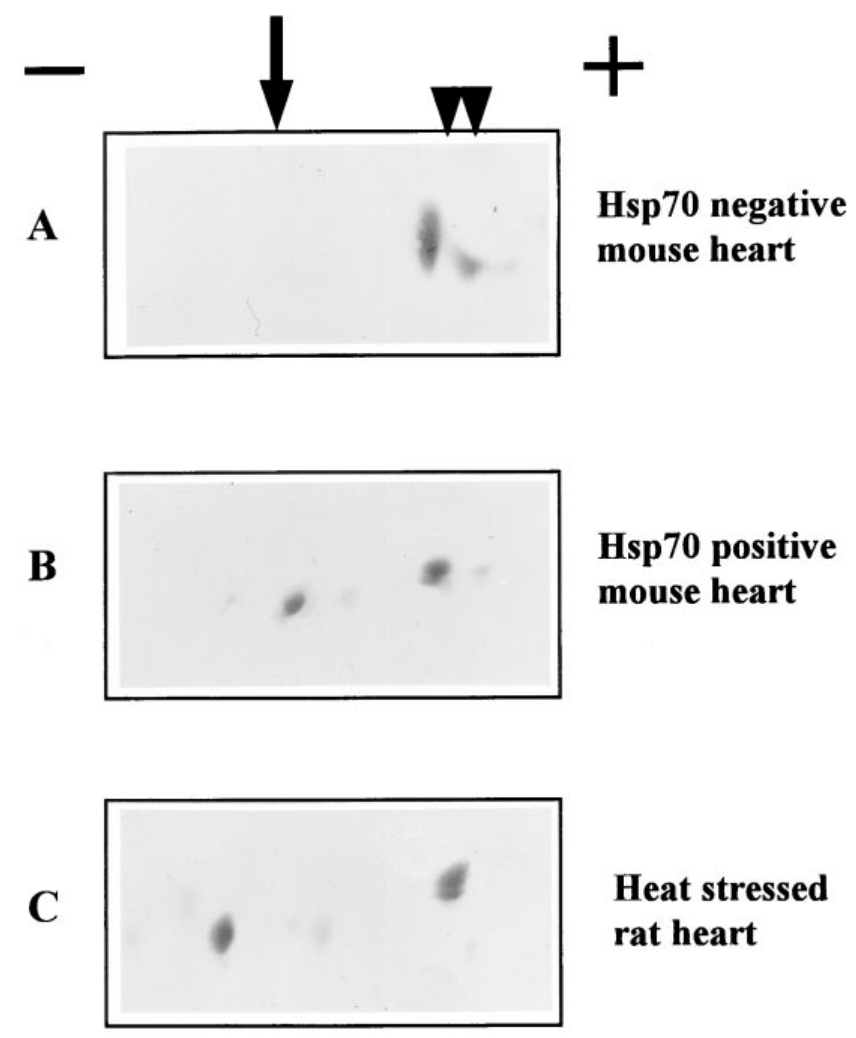

Figure 2. Two-dimensional PAGE Western blot analysis of protein extracts from heart tissue. Protein extracts from hearts of a transgene-negative mouse $(A)$, an HSP70-positive mouse $(B)$ and from a heat-stressed rat $(C)$ were separated by two-dimensional electrophoresis. The presence or absence of the different isoforms of HSP70 were detected by Western blot analysis. Arrowheads indicate the position of the constitutive isoforms of HSP70. The arrow indicates the position of the inducible isoform of HSP70.

representative blots in Fig. 2 show the expression of constitutive HSP70 in a transgene-negative littermate $(A)$ and an HSP70-positive mouse $(B)$. A heart of a heat-shocked rat (23) served as a positive control for expression of inducible HSP70 (C). Rat inducible HSP70 was detected in a transgene-positive mouse at approximately the same isoelectric point compared with the heat-shocked rat, and could not be detected in a negative littermate. The experiment was performed in duplicate.

Hemodynamic measurements. Mean systolic and diastolic arterial pressure in the sham-operated group and in HSP70positive and -negative mice before ischemia was 105/77, 97/76, and $96 / 75 \mathrm{mmHg}$, respectively, and did not differ among the groups (Table I). Over time, systolic and diastolic pressure decreased in the sham-operated group, the ischemic HSP70-positive group, and the ischemic transgene-negative littermates. No statistical differences were found among groups. Blood pressure at the end of the protocol averaged $78 / 52 \mathrm{mmHg}$ in the sham group, $65 / 49 \mathrm{mmHg}$ in the reperfused HSP70-positive group, and $67 / 47 \mathrm{mmHg}$ in the transgene-negative mice. Heart rate showed no statistical differences among the groups, nor did it change over time for the duration of the protocol (Table I).

Regional function in sham-operated mice. Systolic maximum principal strain was used to quantify regional function. This 
Table I. Hemodynamic Results and Principal Strains in HSP70-positive and Transgene-negative Mice

\begin{tabular}{|c|c|c|c|c|c|c|}
\hline & \multicolumn{2}{|c|}{ Sham group } & \multicolumn{2}{|c|}{ HSP 70-positive mice } & \multicolumn{2}{|c|}{ Negative littermates } \\
\hline & Basal & $60 \mathrm{Min}$ & Basal & $60 \mathrm{Min}$ & Basal & $60 \mathrm{Min}$ \\
\hline \multicolumn{7}{|l|}{ Hemodynamic data } \\
\hline Systolic BP (mmHg) & $105 \pm 9$ & $78 \pm 8$ & $97 \pm 6$ & $65 \pm 4$ & $96 \pm 5$ & $67 \pm 5$ \\
\hline Diastolic BP (mmHg) & $77 \pm 8$ & $52 \pm 5$ & $76 \pm 6$ & $49 \pm 4$ & $75 \pm 4$ & $47 \pm 4$ \\
\hline Heart rate (beats/min) & $262 \pm 22$ & $258 \pm 16$ & $257 \pm 13$ & $239 \pm 16$ & $257 \pm 11$ & $280 \pm 11$ \\
\hline \multicolumn{7}{|l|}{ Principal strains } \\
\hline Maximum principal strain $\left(E_{1}\right)$ & $-0.113 \pm 0.014$ & $-0.120 \pm 0.013$ & $-0.110 \pm 0.009$ & $-0.092 \pm 0.005$ & $-0.138 \pm 0.019$ & $-0.071 \pm 0.006$ \\
\hline Angle of $E_{1}$ & $-31 \pm 6^{\circ}$ & $-25 \pm 8^{\circ}$ & $-47 \pm 9^{\circ}$ & $-30 \pm 10^{\circ}$ & $-59 \pm 7^{\circ}$ & $-44 \pm 11^{\circ}$ \\
\hline Minimum principal strain $\left(\mathrm{E}_{2}\right)$ & $-0.020 \pm 0.016$ & $-0.018 \pm 0.010$ & $-0.033 \pm 0.007$ & $-0.035 \pm 0.007$ & $-0.030 \pm 0.006$ & $-0.022 \pm 0.007$ \\
\hline
\end{tabular}

Results of hemodynamic measurements (arterial pressure, heart rate) and of regional function (maximum principal strain $\mathrm{E}_{1}$, minimum principal strain $E_{2}$, angle of $E_{1}$ ) in the in vivo open-chest study. Data are given as mean \pm SEM in sham-operated mice, HSP70-positive mice, and transgene-negative littermates undergoing regional myocardial ischemia at basal time and at $60 \mathrm{~min}$ of reperfusion.

negative strain corresponds to the maximum epicardial segment shortening at peak systole. Fig. 3 shows the individual maximum principal strains for sham-operated mice. Basal mean maximum principal strain $\left(\mathrm{E}_{1}\right)$ was $-0.113 \pm 0.014$ with an angle of $-31 \pm 6^{\circ}$. $E_{1}$ and the angle of $E_{1}$ did not change throughout the experiment (Table I).

Regional function after ischemia and reperfusion. The primary goal of our study was to determine if HSP70 could protect against myocardial dysfunction induced by a brief episode of ischemia not resulting in myocardial infarction. Therefore, we searched for an ischemic period long enough to induce a loss of regional myocardial function, but short enough to avoid myocardial infarction. In preliminary experiments using normal mice, we determined that 15 min of ischemia resulted in myocardial infarcts as indicated by TTC staining. $8 \mathrm{~min}$ of ischemia resulted in a progressive decrease of myocardial function during a 60 -min reperfusion period, whereas after $5 \mathrm{~min}$ of ischemia there was no decline in myocardial function during the following $60 \mathrm{~min}$ of reperfusion (28). Therefore, we chose an ischemic time period of $8 \mathrm{~min}$ in the in vivo open-chest model. Previous studies in isolated mouse hearts showed a functional decrease after a similar ischemic time period (29). Infarction of the myocardium was excluded by TTC staining $(30,16)$. To assess the possibility of false negative results with this method, we stained myocardial tissue from two mice undergoing $45 \mathrm{~min}$ of ischemia and $25 \mathrm{~min}$ of reperfusion. As expected, this tissue stained positive, indicating myocardial infarction in these mice. To further confirm the absence of micro infarctions after 8 min of ischemia, we histologically assessed four additional hearts $5 \mathrm{~d}$ after the ischemic event. No signs of infarctions were found.

Regional myocardial function in both groups of mice as measured by the maximum principal strain $\left(\mathrm{E}_{1}\right)$ after 8 min of LAD occlusion is shown in Fig. $4 A$. Maximum principal strain is plotted as percentage of the basal value. In transgene-negative littermates, a pronounced decrease in function during reperfusion was observed $(P<0.01$, ANOVA), whereas in the HSP70-positive animals the decrease of myocardial function during reperfusion was not statistically significant. After 60 min of reperfusion, HSP70-positive mice had preserved regional function when compared with their transgene negative littermates with $88 \pm 6$ vs. $58 \pm 6 \%$ of the basal values $(P<$ $0.05)$. Absolute values of $E_{1}$ are shown in Table $I$.
Mean angles of $\mathrm{E}_{1}$ were not significantly different at $-47 \pm 9^{\circ} \mathrm{C}$ and $-59 \pm 7^{\circ} \mathrm{C}$ for the HSP70-positive mice and negative littermates, respectively. Fig. $4 B$ shows the mean changes of the principal angle of $E_{1}$ in the two groups during reperfusion. The direction of $\mathrm{E}_{1}$ was stable in both groups throughout the experiment. Basal values of minimum principal strain $\left(E_{2}\right)$ before ischemia and at 60 min of reperfusion are

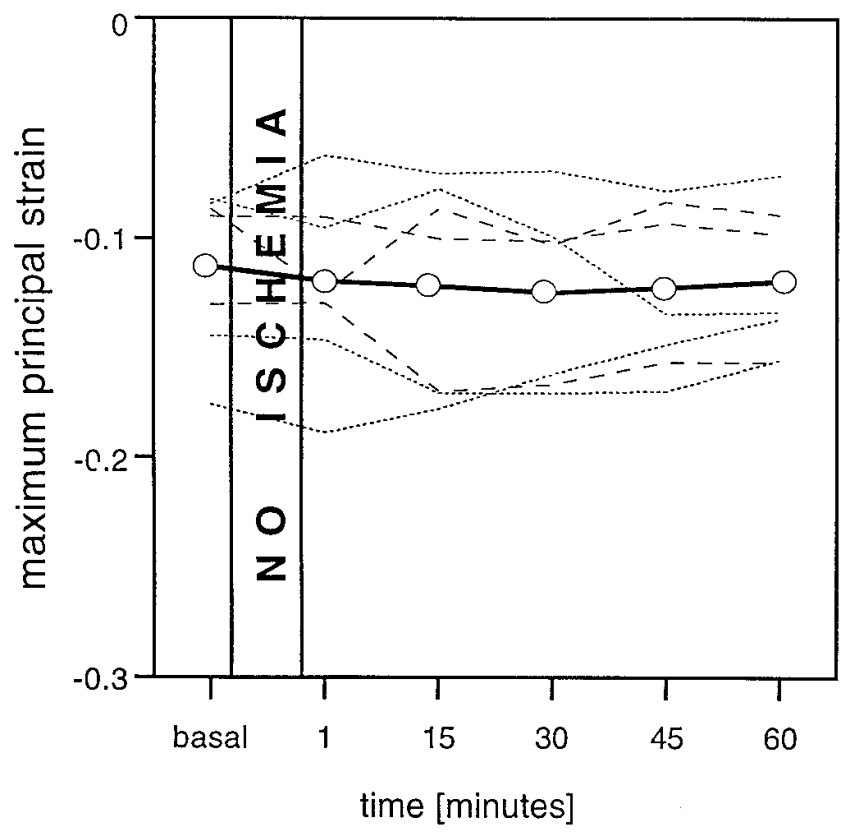

Figure 3. Maximum principal strain $\left(\mathrm{E}_{1}\right)$ in sham-operated mice. The same surgical procedures used in the ischemia protocol were applied to sham-operated mice except for occlusion of the snare around the coronary. $E_{1}$ was measured over a similar time period as in the ischemia protocol. The "no ischemia" bar represents $8 \mathrm{~min}$ corresponding to the time of ischemia, followed by $60 \mathrm{~min}$ analogous to the reperfusion period. $\mathrm{E}_{1}$ was obtained in HSP70-positive mice (dotted line; $n=4)$ and transgene negative littermates (dashed line; $n=3$ ). Strains for the individual animals (fine lines) and the mean strain (solid line) are presented. 
A

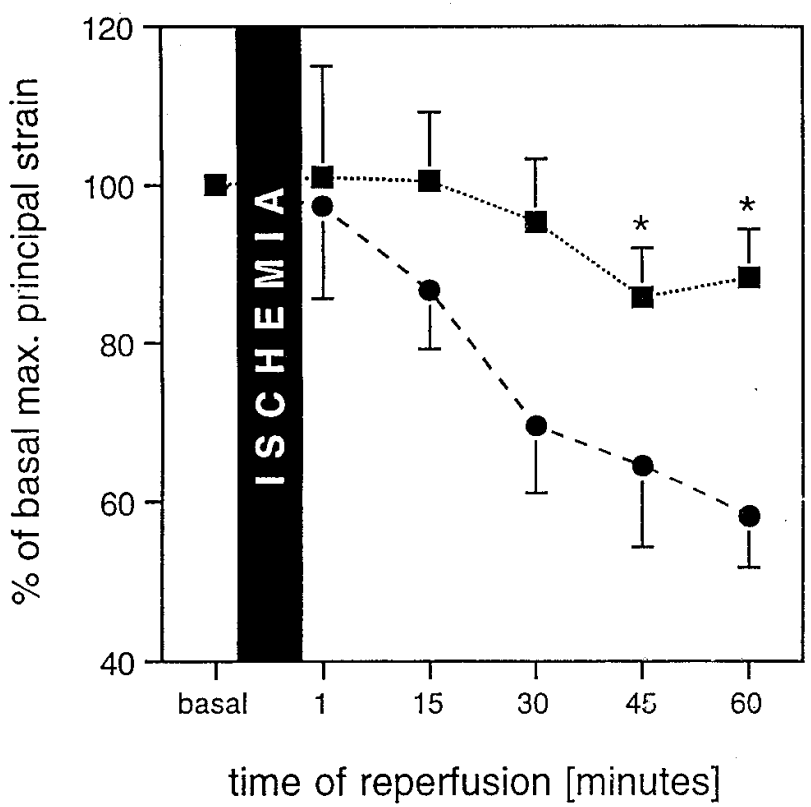

B

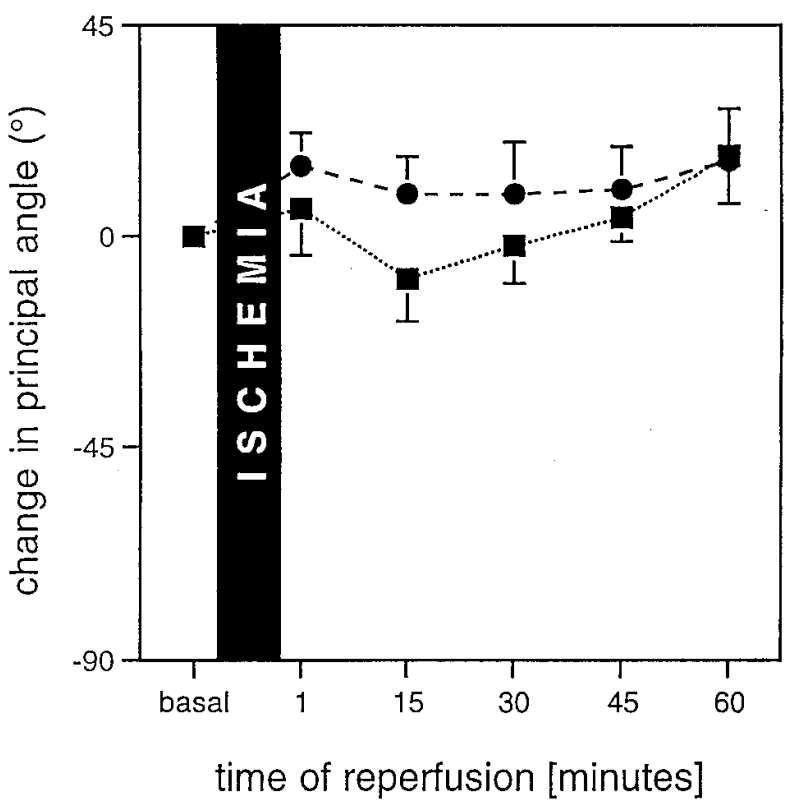

Figure 4. Maximum principal strain $\left(\mathrm{E}_{1}\right)$ and angle in hearts of HSP70-positive and transgene-negative mice undergoing ischemia and reperfusion. $(A) \mathrm{E}_{1}$ was used to measure regional myocardial function in the open-chest preparations. $\mathrm{E}_{1}$ is shown as a percentage of basal value before ischemia in HSP70-positive mice $(n=11)$ and their transgene-negative littermates $(n=11)$. "Ischemia" represents 8 min of occlusion of the left descending coronary artery. The endpoint was $60 \mathrm{~min}$ after start of reperfusion. In HSP70-positive mice, maximum principal strain during reperfusion was significantly better preserved than in the transgene-negative littermates $(P<0.05$, ANOVA). ( $B)$ To demonstrate the stability of the direction of the maximum principal strain throughout the experiments, changes of the angle of $\mathrm{E}_{1}$ from the basal angle before ischemia were calculated. Results are presented as mean \pm SEM. $* P<0.05$ for HSP70-positive mice vs. their transgene-negative littermates. HSP70-positive mice $(\mathbf{\square})$, transgene negative littermates $(\bullet)$.

shown in Table I. Minimum principal strain $\left(\mathrm{E}_{2}\right)$ did not change in either group during the protocol.

Isolated perfused hearts. To evaluate a potential protective effect of HSP70 against postischemic dysfunction by a separate approach, we used a Langendorff isolated heart model. Hearts of HSP70-positive mice $(n=11)$ and their transgene negative littermates $(n=11)$ were exposed to $10 \mathrm{~min}$ of global ischemia and $90 \mathrm{~min}$ of reperfusion. Global myocardial function was determined by measurement of ventricular peak systolic pressure and plotted as percentage of basal value (Fig. 5). Preliminary experiments had indicated that $10 \mathrm{~min}$ of global ischemia was an appropriate time period to induce decreased cardiac function without causing myocardial infarction. In both groups ventricular peak systolic pressure decreased after the onset of reperfusion. Significantly better global function was found in the HSP70-positive mice when compared with their transgene negative littermates $(P<0.05$, ANOVA). In HSP70-positive hearts, peak systolic pressure after 60 and 90 min of reperfusion were not different from the values before ischemia, whereas in the transgene-negative hearts peak systolic pressure was found to be depressed by $72 \pm 7$ and $68 \pm 7 \%$, respectively $(P<0.005)$. Staining of the myocardium at the end of the protocol with TTC showed no evidence of infarction in the heart. Measurement of CK release (Fig. 6) in the effluent of isolated hearts showed no increased enzyme release from hearts exposed to 10-min ischemia when compared with hearts not exposed to ischemia. The dramatically increased
CK release from hearts exposed to 30 -min ischemia at $3 \mathrm{~min}$ $(P<0.05)$ and 30 -min $(P<0.05)$ reperfusion with 2.268土 $0.710 \mathrm{IU} / \mathrm{min} / \mathrm{g}$ heart wt and $1.134 \pm 0.348 \mathrm{iu} / \mathrm{min} / \mathrm{g}$ heart $\mathrm{wt}$ indicate that our CK measurements in the effluent of Langendorff preparations are reliable. Since CK release from the 10 min ischemia group was not different from that of the nonischemic control group, we conclude that $10 \mathrm{~min}$ of global ischemia does not cause significant cardiomyocyte death.

\section{Discussion}

The ability of HSP70 to decrease infarct size in a transgenic HSP70 mouse model has been well-documented $(15,16)$. From these results, we hypothesized that cardiac expression of HSP70 would be sufficient to preserve myocardial function after short periods of ischemia. With increased use of invasive procedures in cardiology and cardiac surgery leading to brief ischemic episodes, development of protective measures against temporary cardiac dysfunction are of importance. Towards this aim, we have shown that expression of inducible HSP70 in hearts of transgenic mice exerts a protective effect against diminished cardiac function induced by a brief period of ischemia not resulting in myocardial infarction. These observations were made in an in vivo model in which regional myocardial function was monitored in an open-chest mouse preparation. Studies in an isolated perfused heart confirmed 


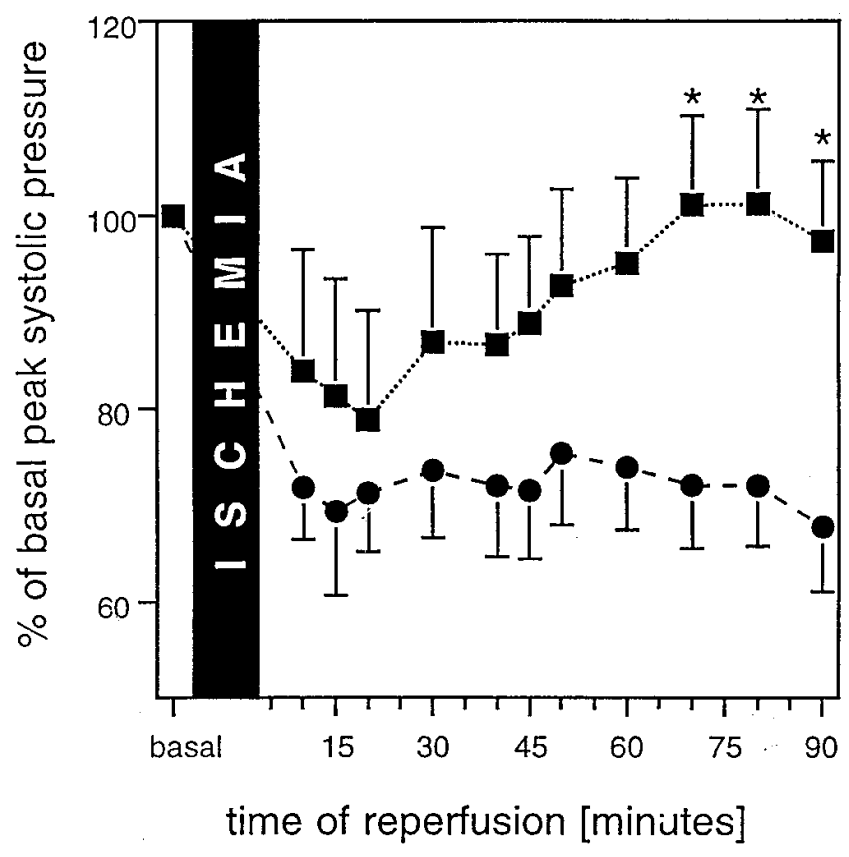

Figure 5. Ventricular peak systolic pressure in isolated Langendorff perfused hearts undergoing ischemia and reperfusion. Peak systolic pressure is shown as a percentage of the preischemic basal value. Hearts from HSP70-positive mice $(\mathbf{\square} ; n=11)$ and their transgenenegative littermates $(\mathbf{\bullet} ; n=11)$ were exposed to $10 \mathrm{~min}$ of ischemia followed by $90 \mathrm{~min}$ of reperfusion. The decrease in ventricular peak systolic pressure during reperfusion was significantly smaller in hearts from HSP70-positive mice than in hearts from their transgene-negative littermates $(P<0.05$, ANOVA). Results are presented as mean \pm SEM. $* P<0.05$ for HSP70-positive mice vs. their transgenenegative littermates.

the protective effect of HSP70 against postischemic dysfunction.

Transgenic mouse hearts expressing specific proteins present interesting models for exploring the influence of transgene products on cardiac function. This exploration requires reliable techniques to examine cardiac function in vivo. A method to quantify regional myocardial contractility in rats was previously established in our laboratory (21). We were able to adapt this method to the smaller mouse heart. The measurements are based on analysis of 2-D epicardial strain by tracking epicardial markers through the cardiac cycle. These markers were spaced close enough together so that a homogenous strain distribution could be assumed in that particular region of the myocardium. The values of maximum principal strain $(-0.138 \pm 0.19)$ were found to be well in accordance with the values $(-0.158 \pm 0.036)$ obtained in a previous study in rats (21). In sham-operated mice, the maximum principal strain $\left(\mathrm{E}_{1}\right)$ did not change during the protocol. Mice undergoing brief ischemia showed a decrease in $E_{1}$ without changes in the angle of $E_{1}$. We concluded that the observed decrease in $E_{1}$ is caused by a depressed myocardial function, and is not due to changes in the direction of principal strain. Measurement of the 2-D epicardial strain has been shown to be a sensitive method to assess changes in regional myocardial function in dogs with myocardial infarction (31) and in stunned rabbit hearts (32). Coronary anatomy in mice was characterized earlier (33), and previous infarct studies with occlusion of the left descending coronary artery showed an area of risk that included most of the anterior wall (16). After each experiment, the area of risk was demarcated with Monastral blue dye to confirm that the chosen titanium oxide markers were well within the ischemic region.

The in vivo results showing a protective effect of HSP70 against decreased myocardial function induced by brief ischemia were confirmed by using a well-established isolated heart approach. A retrograde-perfused Langendorff preparation was used, and ventricular peak systolic pressure was taken as an index for global function. Hearts of HSP70-positive mice showed significantly better function during reperfusion compared with hearts from their transgene-negative littermates. This result indicates that the protective effect seen in vivo is indeed intrinsic to expression of HSP70 in the myocardium. The detailed molecular mechanisms by which HSP70 protects against this type of myocardial dysfunction may be different from mechanisms of protection against myocardial infarction. Ischemic damage takes place in the absence of oxygen, whereas reperfusion damage is thought to be induced predominantly by oxygen-free radicals (17-19). The presence of oxygen-free radicals may contribute to calcium overload (19), protein malfolding (20), and partial protein denaturation leading to a decreased calcium affinity of myofilamental proteins (34). The protective effect of HSP70 against postischemic myocardial dysfunction can be explained with the known chaperone function of members of the HSP70 family $(35,36)$. During re-

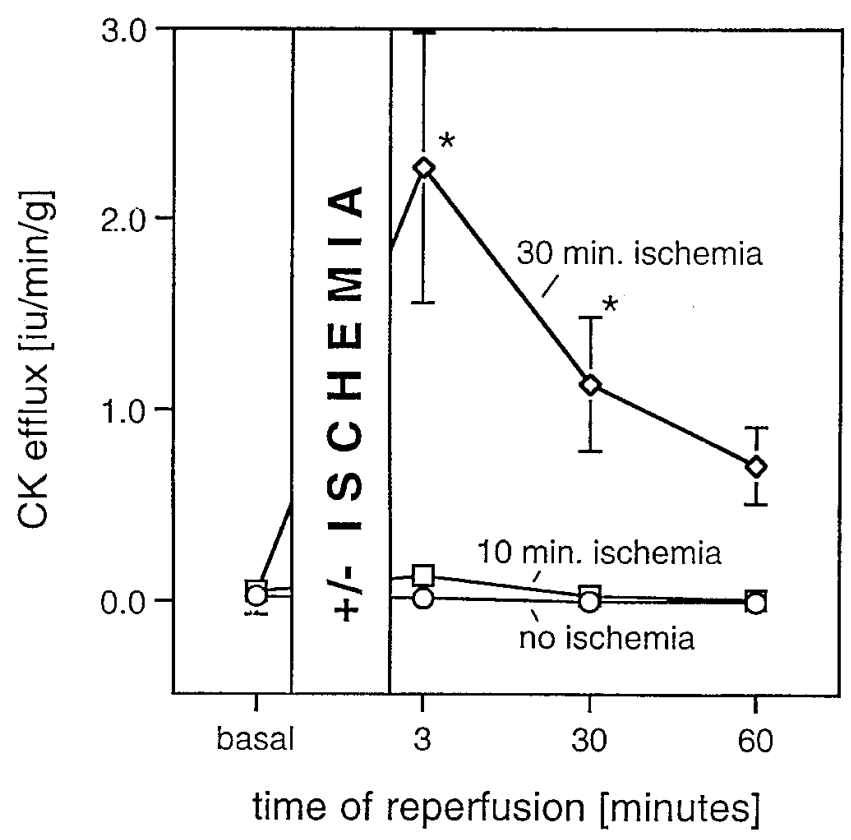

Figure 6. CK release from isolated perfused hearts. CK release in the effluent, which was repetitively collected for $1 \mathrm{~min}$, is shown in IU and corrected for heart weight. Enzyme release from hearts exposed to 10 -min ischemia according to the ischemia protocol $(\square ; n=4)$ did not increase at any time point compared with negative control hearts, which were not exposed to ischemia $(\bigcirc ; n=4)$. Hearts exposed to 30min ischemia $(\diamond ; n=4)$ showed significantly elevated levels of CK release at 3 and $30 \mathrm{~min}$ of reperfusion when compared with control $(P<0.05)$ and to the $10-\mathrm{min}$ ischemia group $(P<0.05)$. 
perfusion, normal protein function and return of protein synthesis to a normal level may occur more rapidly with increased levels of HSP70. Our results show increased protection against myocardial dysfunction after brief ischemia using transgenic mice expressing HSP70 proteins. Such an effect had been postulated in earlier studies using a pig model in which prior brief episodes of ischemia induced HSP70 expression (5). It is appropriate to assume that these maneuvers also have induced other changes, and that the observed protective effect may not be solely attributed to HSP70.

Along with the 2-D strain measurements it was necessary to monitor carefully different functional parameters to ensure the stability of our preparations. From the beginning of this study we found that it was not possible to maintain stable blood pressure in an open-chest mouse. This finding, which is also present in rat open-chest preparations, is most likely due to fluid loss and the side effects of the anesthetic agents (37). Initial attempts to compensate for fluid loss did not increase the stability of blood pressure. However, the decrease in blood pressure was the same in sham-operated and ischemic mice of either transgene status. The inability to observe cardiac function recovery during reperfusion in control mice is a limitation of our study that cannot be overcome in our open-chest model. Since the term "myocardial stunning" includes a recovery phase, our model does not fulfill all the criteria of a stunning model. We therefore avoided using this terminology. Heart rate in all groups was slightly below the values described in conscious mice (24), but remained stable during the experiments. The cardiovascular parameters in this preparation were still sufficient to maintain the viability of myocardial tissue as no myocardial infarction developed in mice examined $5 \mathrm{~d}$ after surgery.

Increased expression of inducible HSP70 in transgenic mice protects myocardial function from reperfusion damage after brief ischemic periods not resulting in infarction. Transgene-positive mice and their negative littermates showed no other difference in cardiovascular function during reperfusion. The protective effect against postischemic myocardial dysfunction was documented in an open-chest in vivo model using a newly developed method to measure regional myocardial function in the mouse heart. The protective effect of HSP70 against postischemic myocardial dysfunction was confirmed ex vivo in an isolated perfused Langendorff heart model. Thus, we can conclude that isolated expression of inducible HSP70 in the myocardium is sufficient to protect from myocardial dysfunction induced by brief ischemic periods.

\section{Acknowledgments}

We wish to thank T. Wang and A. Brown for help on data processing, R. Pavelec for technical assistance, and Dr. H.A. Rockman for his advice on the in vivo mouse preparation.

This work was supported in part by the National Institutes of Health R01 grants HL49434, and HL32583 and a grant from the American Heart Association CA96-275.

\section{References}

1. Heyndrickx, G.R., R.W. Millard, R.J. McRitchie, P.R. Maroko, and S.F. Vatner. 1975. Regional myocardial function and electrophysiological alterations after brief coronary artery occlusion in conscious dogs. J. Clin. Invest. 56: 978-985.

2. Braunwald, E., and R. Kloner. 1982. The stunned myocardium: pro- longed, postischemic ventricular dysfunction. Circulation. 66:1146-1149.

3. Bolli, R. 1990. Mechanisms of myocardial stunning. Circulation. 82:723-738.

4. Patel, B., R. Kloner, K. Przyklenk, and E. Braunwald. 1988. Postischemic myocardial "stunning": a clinically relevant phenomenon. Ann. Intern. Med. 108:626-628.

5. Sun, J., X. Tang, A.A. Knowlton, S. Park, Y. Qiu, and R. Bolli. 1995. Late preconditioning against myocardial stunning. J. Clin. Invest. 95:388-403.

6. Dillmann, W.H., H.B. Mehta, A. Barrieux, B.D. Guth, W.E. Neeley, Jr., and J. Ross, Jr. 1986. Ischemia of the dog heart induces the appearance of a cardiac mRNA coding for a protein with migration characteristics similar to heatshock/stress protein 71. Circ. Res. 59:110-114.

7. Mehta, H.B., B.K. Popovich, and W.H. Dillmann. 1988. Ischemia induces changes in the level of mRNAs coding for stress protein 71 and creatine kinase M. Circ. Res. 63:512-517.

8. Knowlton, A., P. Brecher, and C. Apstein. 1991. Rapid expression of heat shock protein in the rabbit after brief cardiac ischemia. J. Clin. Invest. 97:139-147.

9. Currie, R. 1987. Effects of ischemia and reperfusion temperature on the synthesis of stress-induced (heat shock) proteins in isolated and perfused hearts. J. Mol. Cell. Cardiol. 19:795-808.

10. Donnelly, T.J., R.E. Sievers, F.L.J. Vissern, W.J. Welch, and C.L. Wolfe. 1992. Heat shock protein induction in rat hearts. Circulation. 85:769-778.

11. Locke, M., R.M. Tanguay, R.E. Klabunde, and C.D. Ianuzzo. 1995. Enhanced postischemic myocardial recovery following exercise induction of HSP72. Am. J. Physiol. 269:H320-H325.

12. Currie, R.W., M. Karmazyn, M. Kloc, and K. Mailer. 1988. Heat-shock response is associated with enhanced postischemic ventricular recovery. Circ. Res. 63:543-549.

13. Hoshida, S., T. Kuzuya, H. Fuji, N. Yamashita, H. Oe, M. Hori, K. Suzuki, N. Taniguchi, and M. Tada. 1993. Sublethal ischemia alters myocardial antioxidant activity in canine heart. Am. J. Physiol. 264:H33-H39.

14. Mestril, R., and W.H. Dillmann. 1991. Heat shock and adaptive response to ischemia. Trends Cardiovasc. Med. 1:240-244.

15. Marber, M., R. Mestril, S.H. Chi, M.R. Sayen, D.M. Yellon, and W.H. Dillmann. 1995. Overexpression of the rat inducible 70-kD heat stress protein in a transgenic mouse increases the resistance of the heart to ischemic injury. $J$. Clin. Invest. 95:1146-1456.

16. Hutter, J.J., R. Mestril, E.K.W. Tam, R.E. Sievers, W.H. Dillmann, and C.L. Wolfe. 1996. Overexpression of inducible heat shock protein (HSP) 72 in transgenic mice decreases infarct size. Circulation. 94:1408-1411.

17. Li, X., P.B. McCay, M. Zughaib, M.O. Jeroudi, J.F. Triana, and R. Bolli. 1993. Demonstration of free radical generation in the "stunned" myocardium in the conscious dog and identification of major differences between conscious and open-chest dogs. J. Clin. Invest. 92:1025-1041.

18. Opie, L.H. 1989. Reperfusion injury and its pharmacologic modification. Circulation. 80:1049-1061.

19. Hess, M.L., and R.C. Kukreja. 1995. Free radicals, calcium homeostasis, heat shock proteins, and myocardial stunning. Ann. Thorac. Surg. 60:760-766.

20. Nguyen, V.T., M. Morange, and O. Bensaude. 1989. Protein denaturation during heat shock and related stress. J. Biol. Chem. 264:10487-10492.

21. Omens, J.H., D.D. Farr, A.D. McCulloch, and L.K. Waldman. 1996. Comparison of two techniques for measuring two-dimensional strain in the rat left ventricle. Am. J. Physiol. 271:H1256-H1261.

22. Villareal, F.J., L.K. Waldman, and W.Y.W. Lew. 1988. Technique for measuring regional two-dimensional finite strains in canine left ventricle. Circ. Res. 62:711-721.

23. Mestril, R., S.H. Chi, M.R. Sayen, and W.H. Dillmann. 1994. Isolation of a novel inducible rat heat-shock protein (HSP70) gene and its expression during ischemia/hypoxia and heat shock. Biochem. J. 298:561-569.

24. Milano, C.A., L.F. Allen, H.A. Rockman, P.C. Dolber, T.R. McMinn, K.R. Chien, T.D. Johnson, R.A. Bond, and R.J. Lefkowitz. 1994. Enhanced myocardial function in transgenic mice overexpressing the $\mathrm{B}_{2}$-adrenergic receptor. Science. 264:582-585.

25. Grupp, I., and G. Grupp. Isolated heart preparations perfused or superfused with balanced salt solutions. 1984. In Methods of Pharmacology. A Schwartz, editor. Plenum Publishing Corp, New York. 111-128.

26. Neely, J., H. Liebermeister, E. Battersby, and H. Morgan. 1967. Effect of pressure development on oxygen consumption by isolated rat heart. Am. J. Physiol. 212:804-814.

27. Marcus, R., E. Peritz, and K.R. Gabriel. 1976. On closed testing procedures with special reference to ordered analysis of variances. Biometrica. 63 655-660.

28. Trost, S.U., J.H. Omens, W.J. Karlon, W.H. Dillmann, and J.W. Covell. 1996. Regional epicardial strain during myocardial stunning in the mouse. Circulation. 94(Abstr.):I-185.

29. Manche, A., S.J. Edmondson, and D.J. Hearse. 1994. Dynamics of early postischemic myocardial recovery. Circulation. 92:526-564.

30. Ganz, W.W.I., K. Kanamasa, J. Yano, D.S. Han, and M.C. Fishbein 1990. Does reperfusion extend necrosis? Circulation. 82:1020-1033.

31. Villarreal, F.J., L.K. Waldman, and J.W. Covell. 1991. Transmural myocardial deformation in the ischemic canine left ventricle. Circ. Res. 68:368-381.

32. Lew, W.Y.W., Y. Nishikawa, and H. Su. 1994. Cardiac myocyte function and left ventricular strains after brief ischemia and reperfusion in rabbits. Cir- 
culation. 90:1942-1950.

33. Michael, L.H.E., C.J. Hartley, K.A. Youker, J. Zhu, S.R. Hall, H.K. Hawkins, K. Berens, and C.M. Ballentyne. 1995. Myocardial ischemia and reperfusion: a murine model. Am. J. Physiol. 269:H2147-H2154.

34. Gao, W.D., D. Atar, P.H. Backx, and E. Marban. 1995. Relationship between intracellular calcium and contractile force in stunned myocardium. Circ. Res. 76:1036-1048.
35. Ellis, J. 1987. Proteins as molecular chaperones. Nature. 328:378-379.

36. Beckmann, R., and W.J.W. Lam. 1990. Interaction of Hsp70 with newly synthesized proteins: implications for protein folding and assembly. Science. 248:248-253.

37. Ji, L.L., R.G. Fu, T.G. Waldrop, K.J. Liu, and H.M. Swartz. 1993. Myocardial response to regional ischemia and reperfusion in vivo in rat heart. Can J. Physiol. Pharamcol. 71:811-817. 